\title{
Evaluating the control of HPAIV H5N1 in Vietnam: virus transmission within infected flocks reported before and after vaccination
}

\author{
Ricardo J Soares Magalhães ${ }^{* 1,2}$, Dirk U Pfeiffer ${ }^{1}$ and Joachim Otte ${ }^{3}$
}

\begin{abstract}
Background: Currently, the highly pathogenic avian influenza virus (HPAIV) of the subtype H5N1 is believed to have reached an endemic cycle in Vietnam. We used routine surveillance data on HPAIV H5N1 poultry outbreaks in Vietnam to estimate and compare the within-flock reproductive number of infection $\left(R_{0}\right)$ for periods before (second epidemic wave, 2004-5; depopulation-based disease control) and during (fourth epidemic wave, beginning 2007; vaccinationbased disease control) vaccination.

Results: Our results show that infected premises (IPS) in the initial (exponential) phases of outbreak periods have the highest $R_{0}$ estimates. The IPs reported during the outbreak period when depopulation-based disease control was implemented had higher $R_{0}$ estimates than IPs reported during the outbreak period when vaccination-based disease control was used. In the latter period, in some flocks of a defined size and species composition, within-flock transmission estimates were not significantly below the threshold for transmission $\left(R_{0}<1\right)$.

Conclusions: Our results indicate that the current control policy based on depopulation plus vaccination has protected the majority of poultry flocks against infection. However, in some flocks the determinants associated with suboptimal protection need to be further investigated as these may explain the current pattern of infection in animal and human populations.
\end{abstract}

\section{Background}

The incidence of HPAIV H5N1 cases in poultry and humans has been particularly high in south-east Asian countries and Egypt [1]. The current pattern of poultry outbreaks in these regions suggests the presence of a reservoir of residual infection $[2,3]$.

From late 2003 to 2006 in Vietnam, the incidence of outbreaks in poultry was particularly high in regions of the Red and Mekong river deltas. Outbreaks were associated with increased movement of poultry around the annual traditional festivities held just before and during February (the "Tet" holiday) [4]. However, since 2006, this pattern has discontinued. Across the country, HPAIV H5N1 infection primarily affected small-scale commercial premises rearing chicken or ducks in subsistence

*Correspondence: r.magalhaes@sph.uq.edu.au

1 The Royal Veterinary College, Department of Veterinary Clinical Sciences, Veterinary Epidemiology and Public Health Group, London, UK, Hawkshead Lane AL9 7TA, UK

Full list of author information is available at the end of the article flocks $[5,6]$. These market-oriented flocks account for about two-thirds of poultry production and half of direct marketing in Vietnam $[7,8]$. In addition to poultry cases, Vietnam has the second highest number of HPAIV H5N1-related human fatalities in the world [9]. Between 2003 and May 2010, there were 119 confirmed HPAIV H5N1 cases and 59 deaths in humans, representing a case-fatality rate of 49.6 percent.

In the early stages of the epidemic, the institutional responses in affected areas throughout Vietnam were administratively and temporally inconsistent. Measures included various large-scale depopulation (stamping-out) policies, and restrictions on poultry movement, breeding of certain poultry species, and the sale of live poultry in wet markets [10]. In September 2005, the Department of Animal Health of Vietnam started HPAIV H5N1 vaccination campaigns of susceptible poultry flocks. These campaigns were heterogeneous in terms of the timing of 
spatial coverage, the vaccine types used and the poultry species targeted [11].

An assessment of the efficacy of disease control measures is essential for guiding future policy. The reproductive number of infection, $R_{0}$, is an averaged epidemiological property of a randomly mixing population with complete susceptibility to the infectious agent and represents the number of secondary infectious cases produced by a typical infectious case during its entire life expectancy [12]. The $R_{0}$ measures the transmissibility of an infectious agent in a given host population and thus is a sensitive indicator of the impact of disease control interventions applied at flock and herd level [13]. The estimation and evaluation of within-flock transmissibility of avian influenza viruses is important, as this influences the flock-to-flock transmissibility and enables the development of predictive models of large-scale disease control interventions [14-17].

Few studies have assessed the efficacy of disease control efforts in the face of HPAIV H5N1 outbreaks by estimating the within-flock $R_{0}$ [18]. Instead, either within-flock transmission in industrial-type IPs or in those not subject to vaccination has been quantified. Our study aims to estimate the transmissibility of HPAIV H5N1 in a sample of affected flocks in Vietnam. In doing so, we compared estimates for outbreak periods before and during the systematic vaccination campaigns, for different phases within each of the outbreak waves, and for flocks differing in size and species composition.

\section{Results}

\section{HPAIV H5N1-infected IPs}

Figure 1 shows the time course of the number of IPs for the two outbreak periods considered in the analysis.
Using log-transformed daily incidence of reportedinfected flocks we found for Period I that the exponential growth of the epidemic had reached its peak on 17 January 2005 (data not shown). One week later incidence reached a second peak with duration of 13 days.

A summary of IP characteristics regarding population sizes and observed mortality during both outbreak periods is presented in Table 1. During the two outbreak periods most IPs were duck flocks containing less than 1,500 head of poultry ( $88 \%$ and $92 \%$ respectively) located in the southern part of the country. The proportion of IPs with less than 50 head of poultry (i.e. backyard IPs) was $9 \%$ (93/1005) for Period I and 30\% (34/114) for Period II. In addition, most mixed poultry species IPs (i.e. $92 \%$ for Period I and all in Period II) had less than 1,000 head.

Across both waves, within-flock mortality was found to be significantly higher in chicken IPs with more than 1,000 head, in duck IPs with more than 500 head and in mixed poultry species IPs with more than 3,000 head $(P<$ $0.05)$ than in chicken IPs less than 50 head. There was a marginally significant reduction in within-flock mortality in Period II compared with Period I $(P=0.057)$.

\section{Estimates of within-flock virus transmission}

Figure 1 shows the temporal progression of the estimates of within-flock $R_{0}$ for the outbreak periods considered in the analysis. Our results indicated that, for Period I, IPs in the initial 17 days of the epidemic (1st of January to the 17th of January; exponential growth) had above average within-flock transmission estimates (Figure 1a). During that period, chicken IPs of farm sizes larger than 1,500 head and duck IPs of less than 50 head had higher withinflock $R_{0}$ than chicken IPs of less than 50 head of poultry. In addition, our analysis suggested a second peak of within-flock transmission occurred in the first week of
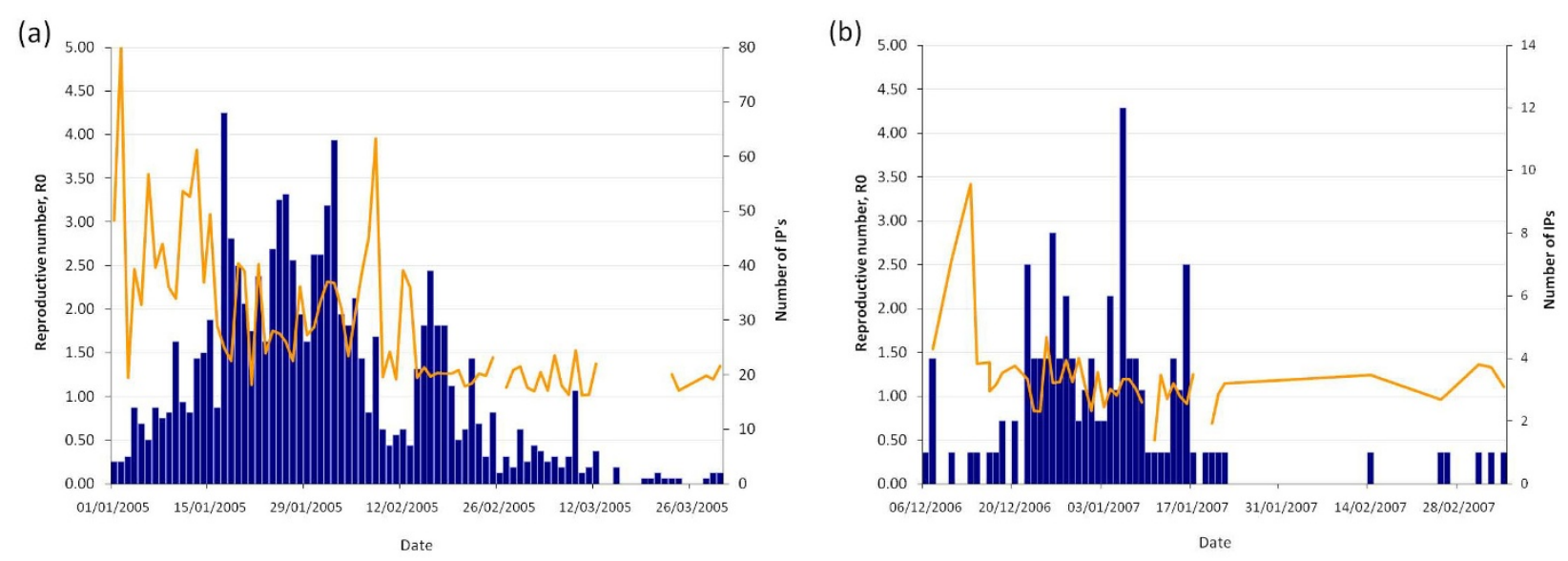

Figure 1 Temporal progression of the newly reported infected premises (IPs) (blue bars) and 3-day moving average of the daily within-

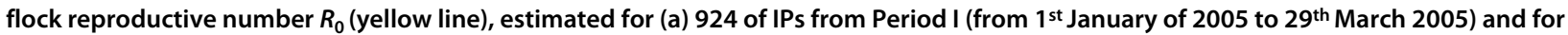
(b) 106 IPs from Period II (from $6^{\text {th }}$ December 2006 to $7^{\text {th }}$ March 2007) in Vietnam. 
Table 1: Number of reported HPAIV H5N1 infected premises (IPs) by flock size and flock mortality categories for Period I (i.e. pre-vaccination period) and Period II (i.e. post-vaccination period) outbreak periods.

\begin{tabular}{|c|c|c|c|c|c|c|c|c|}
\hline & \multicolumn{4}{|c|}{ Pre vaccination IPs } & \multicolumn{4}{|c|}{ Post vaccination IPs } \\
\hline & Chicken & Duck & Mixed & Other & Chicken & Duck & Mixed & Other \\
\hline Total number(proportion) & $318(0.32)$ & $577(0.57)$ & $72(0.07)$ & $38(0.04)$ & $13(0.11)$ & $91(0.8)$ & $4(0.04)$ & $6(0.05)$ \\
\hline \multicolumn{9}{|c|}{ No. of birds initially at risk(proportion) } \\
\hline 1-50 head & $58(0.18)$ & $27(0.05)$ & $8(0.11)$ & 0 & $7(0.54)$ & $18(0.2)$ & $3(0.75)$ & $6(1)$ \\
\hline 51-200 head & $77(0.24)$ & $96(0.17)$ & $25(0.35)$ & $1(0.03)$ & $2(0.15)$ & $23(0.25)$ & 0 & 0 \\
\hline 201-500 head & $74(0.23)$ & $152(0.26)$ & $22(0.31)$ & 0 & 0 & $18(0.2)$ & $1(0.25)$ & 0 \\
\hline $501-1,000$ head & $46(0.14)$ & $154(0.27)$ & $11(0.15)$ & 0 & $1(0.08)$ & $18(0.2)$ & 0 & 0 \\
\hline $1,001-1,500$ head & $21(0.07)$ & $76(0.13)$ & $1(0.01)$ & $1(0.03)$ & $2(0.15)$ & $7(0.08)$ & 0 & 0 \\
\hline 1,501-3,000 head & $21(0.07)$ & $55(0.10)$ & $1(0.01)$ & $6(0.16)$ & 0 & $6(0.07)$ & 0 & 0 \\
\hline 3,001-6,000 head & $15(0.05)$ & $13(0.02)$ & $2(0.03)$ & $10(0.26)$ & 0 & $1(0.01)$ & 0 & 0 \\
\hline$>6,000$ heads & $6(0.02)$ & $4(0.01)$ & $2(0.03)$ & $20(0.53)$ & $1(0.08)$ & 0 & 0 & 0 \\
\hline \multicolumn{9}{|c|}{ No. of birds reported dead (proportion) } \\
\hline 1-20 head & $58(0.18)$ & $37(0.06)$ & $9(0.13)$ & 0 & $9(0.69)$ & $25(0.27)$ & $4(1)$ & $6(1)$ \\
\hline 21-50 head & $67(0.21)$ & $82(0.14)$ & $20(0.28)$ & $1(0.03)$ & $1(0.08)$ & $21(0.23)$ & 0 & 0 \\
\hline $51-150$ head & $71(0.22)$ & $145(0.25)$ & $24(0.33)$ & 0 & 0 & $16(0.18)$ & 0 & 0 \\
\hline $151-300$ head & $50(0.16)$ & $123(0.21)$ & $6(0.08)$ & $10(0.26)$ & 0 & $12(0.13)$ & 0 & 0 \\
\hline 301-500 head & $36(0.11)$ & $87(0.15)$ & $5(0.07)$ & $4(0.11)$ & $1(0.08)$ & $6(0.07)$ & 0 & 0 \\
\hline 501-1,000 head & $18(0.06)$ & $63(0.11)$ & $5(0.07)$ & $5(0.13)$ & $1(0.08)$ & $7(0.08)$ & 0 & 0 \\
\hline$>1,001$ head & $18(0.06)$ & $40(0.07)$ & $3(0.04)$ & $18(0.47)$ & $1(0.08)$ & $4(0.04)$ & 0 & 0 \\
\hline
\end{tabular}

February. A similar temporal pattern of within-flock $R_{0}$ was found during Period II (Figure 1b), where IPs reported at the beginning of the epidemic had higher within-flock transmission estimates than subsequent IPs. The highest within-flock $R_{0}$ in the early stages of Period II (also during the exponential growth phase of the epidemic) was estimated in duck IPs of 200 to 1,500 head of poultry followed less than a week later by chicken IPs of less than 50 head of poultry. Across the remainder of Period II, the within-flock $R_{0}$ of IPs of different poultry species and size composition did not differ significantly and was significantly above unity $(P<0.05)$.

The distributions of the within-flock $R_{0}$ estimates for both outbreak periods examined are summarized in Table 2. Our results for Period I showed that mean within-flock $R_{0}$ did not significantly differ between IPs of 
different species composition $(P>0.05)$. During Period II, the mean within-flock $R_{0}$ was estimated to be significantly higher in duck IPs of flock sizes between 50 and 200 and between 500 and 1,500 head of poultry compared to duck flocks of sizes less than 50 head $(P<0.05)$. Comparisons between outbreak periods indicated that the mean within-flock $R_{0}$ was significantly lower during Period II when compared to Period I (95\%CI: -1.16, -0.48; $P<0.05)$. There was no such difference between both outbreak periods for mixed poultry species IPs and for duck IPs with flock sizes above 500 head of poultry $(P>$ $0.05)$. However, the mean estimate of within-flock $R_{0}$ for Period II was still significantly above the threshold of transmission $\left(R_{0}>1\right)(P<0.001)$. The estimated withinflock $R_{0}$ in duck IPs less than 50 head $\left(R_{0}=0.96\right)$ for Period II was not significantly below the threshold of transmission $(P=0.635)$.

\section{Univariable analysis}

We investigated the strength of association between the outcome variables a) within-flock $R_{0}$ on a continuous scale (Model 1) and $\mathrm{b}$ ) within-flock $R_{0}$ estimates categorised as lower than unity or not (Model 2) and three flocklevel risk factors (i.e. "flock species", "flock size" and "epidemic wave"). In the univariable analyses all variables reached the decision criterion for variable selection except "flock species" for Model 2 (Table 3).

\section{Multivariable analysis}

In Model 1, only the variable "epidemic wave" retained significance at a $P<0.05$. Our results suggest that the within-flock $R_{0}$ estimates for Period II are significantly lower compared with Period I. The coefficient of determination of the final model Model 1 was $\mathrm{R}^{2}=0.41$. In Model 2, the variables "flock size" and "epidemic wave" retained significance at a $P<0.05$ (Table 4 ). Our results suggest that after controlling for the effect of flock size, IPs of Period II had increased odds of having within-flock $R_{0}$ below unity compared to IPs of Period I. The goodness-of-fit of the final multivariable model to the data, as assessed by the Hosmer-Lemeshow goodness of fit test, was adequate $(P=0.816)$.

\section{Discussion}

This study compared and characterized the transmissibility of HPAIV H5N1 in affected flocks in Vietnam, before and after the introduction of a control policy that included vaccination. Our results show that measuring within-flock virus transmissibility can improve understanding of the epidemiology and effectiveness of control strategies against HPAIV H5N1 infection in endemic countries.
Control policies based on flock depopulation (such as in Period I) are expected to have a direct impact on the effective within-flock contact rate while control involving depopulation plus preventive vaccination of flocks (such as in Period II) will contribute with the added impact on the properties of the natural history of infection. However, factors such as delays in disease reporting and operational constraints of stamping-out interventions will influence the effectiveness of both control strategies. This circumstance allows the generation of chains of infection within a flock that would not have happened if birds had been stamped-out at an earlier stage. In contrast, vaccination for HPAIV H5N1 is expected to have an individual (direct) effect (e.g. increase the incubation period, reduce the number of infectious virus excreted as well as reduce the duration of infectiousness) and a population (indirect) effect (e.g. via herd immunity) on the natural history of infection [19]. Any partially immune individual introduced infectious into an unvaccinated flock will shed less HPAIV H5N1 particles and be infectious for a shorter period [20,21]. Similarly, the population effect of a vaccination programme that reaches flocks in the vicinity or those that are part of the trade relationships of an unvaccinated flock will gradually contribute to herd immunity (despite the fact that some transmissions may still occur) [22]. In addition, vaccination is expected to contribute to a reduced environmental contamination.

Our study findings suggest a differential effect of disease control interventions on within-flock virus transmissibility. Inspection of the temporal progression of estimates of within-flock $R_{0}$ indicates that IPs of different flock size and species composition are likely to have had different roles in the transmission dynamics during different phases of the outbreak periods examined in our study. Our results show that the within-flock $R_{0}$ of flocks with similar species and size composition varied over the course of each epidemic wave, and higher estimates were found in earlier phases of each epidemic wave. Based on the assumption of constant contact structure and transmission dynamics among flocks of similar species and size composition, this variation may be due to the effect of disease control interventions. The highest within-flock $R_{0}$ observed in the initial phases of the outbreak periods may be attributed to the fact that flocks with high effective contact rates and those that experienced delayed application of control interventions (either by delayed disease notification or operational delays) were disproportionally represented. Our results indicate that chicken IPs with flock size larger than 1,500 head had the highest $R_{0}$ estimates during the initial (exponential) phase of Period I. Previous studies have shown that HPAIV H5N1 spreads more rapidly both in farms with larger numbers of chicken houses in use and among layer chickens (com- 
Table 2: Within-flock reproductive ratio $\left(R_{0}\right)$ estimated in 924 IPs reported during Period I (i.e. pre-vaccination period) and in 106 IPs reported during Period II (i.e. vaccination period).

\section{Infected Premise Species/Size}

\section{Reproductive number, $\boldsymbol{R}_{0}$ (Mean; $95 \% \mathrm{Cl}$; $\mathrm{N}$ )}

\section{Post-vaccination IPs}

$1.17 ; 1.09-1.25 ; 106$

\section{All IP's}

$1.99 ; 1.87,2.10 ; 924$

\section{Chicken flocks only}

Pooled

1-50 heads

51-200 heads

201-500 heads

501-1,000 heads

$1,001-1,500$ heads

$>1,500$ heads

Duck flocks only
$2.16 ; 1.94,2.39 ; 297$

$2.03 ; 1.71,2.36 ; 58$

$1.90 ; 1.55,2.25 ; 77$

$2.28 ; 1.8,2.74 ; 74$

$1.92 ; 1.33,2.50 ; 46$

$2.77 ; 1.45,4.09 ; 21$

$2.86 ; 1.40,4.31 ; 21$

$1.96 ; 1.81,2.11 ; 560$

$2.02 ; 1.53,2.50 ; 27$

$1.85 ; 1.55,2.14 ; 96$

$2.16 ; 1.84,2.48 ; 152$

$1.90 ; 1.61,2.19 ; 154$

$1.92 ; 1.48,2.36 ; 76$

$1.55 ; 1.18,1.92 ; 55$

$>1,500$ heads

\section{Mixed poultry species}

Pooled

$1.91 ; 1.53,2.29 ; 67$

$3.20 ; 2.19,4.21 ; 8$

1-50 heads

51-200 heads

201-500 heads

501-1,000 heads

1,001-1,500 heads
$1.07 ; 0.90,1.23 ; 12$

$1.04 ; 0.76,1.32 ; 7$

$0.80 ; 0.68,1.11 ; 2$

NA

$1.02 ; 0.87,1.24 ; 1$

$1.34 ; 1.03,1.71 ; 2$

NA 
Table 3: Univariable results: within-flock $R_{0}$ vs. flock attributes.

\begin{tabular}{lccc}
\hline Variable & Model 1 & Model 2 \\
\hline & Coefficient $(95 \% \mathrm{Cl})$ & $\boldsymbol{P}$ & OR (95\%Cl) \\
\hline Flock species (Ref: Duck flocks) & & & \\
\hline Mixed poultry species & $0.006(-0.416,0.428)$ & 0.977 & $2.054(0.957,4.411)$ \\
\hline Chicken flocks & $0.264(0.030,0.498)$ & 0.027 & $1.580(0.972,2.570)$ \\
\hline Flock Size(Ref: $<50$ head) & & & 0.065 \\
\hline$>50-500$ head & $0.107(-0.242,0.456)$ & 0.549 & $0.165(0.096,0.286)$ \\
\hline$>500-1,500$ head & $0.111(-0.254,0.476)$ & 0.551 & 0.065 \\
\hline$>1,500$ head & $0.064(-0.351,0.479)$ & 0.762 & $0.160(0.073,0.348)$ \\
\hline
\end{tabular}

Epidemic wave(Ref: Period I)

\begin{tabular}{lllll}
\hline Period II & $-0.821(-1.163,-0.478)$ & $<0.001$ & $4.504(2.689,7.544)$ & $<0.001$ \\
\hline
\end{tabular}

OR: Odds Ratio; SE: Standard Error; Cl: Confidence Interval

pared with broilers) [23]. Similarly, IPs with flock sizes less than 50 head, and duck flocks of 200 to 1,500 head were also identified to have the highest within-flock $R_{0}$ estimates during the initial phases of Period II. Consequently, it is likely that these flocks could have contributed to localised spread of infection observed during Period II - they are characterised by a free-ranging husbandry system and mortality often remains unnoticed and/or unreported mainly due to the absence of records

Table 4: Multivariable results for Model 2: within-flock $R_{0}$ being below unity vs. flock attributes.

\begin{tabular}{lcc}
\hline Variable & OR $(95 \% \mathrm{Cl})$ & $\boldsymbol{P}$ \\
\hline Flock Size $($ Ref: $<50$ head) & & \\
\hline$>50-500$ head & $0.202(0.115,0.355)$ & $<0.001$ \\
\hline$>500-1,500$ head & $0.134(0.067,0.269)$ & $<0.001$ \\
\hline$>1,500$ head & $0.209(0.094,0.464)$ & $<0.001$ \\
\hline
\end{tabular}

Epidemic wave (Ref: Period I)

\begin{tabular}{lrl}
\hline Period II & $3.114(1.785,5.432)$ & $<0.001$ \\
\hline OR: Odds Ratio; SE: Standard Error; Cl: Confidence Interval &
\end{tabular}

and difficulties in monitoring [6]. In addition, it has been suggested that the quality of the depopulation policy between and within each of the outbreak periods was inconsistent (see section\#3 in Additional file 1). Therefore, it is also likely that IPs in the initial phases of the outbreak periods - due to operational constraints resulting from having to deal with a large number of outbreaks simultaneously - had experienced delays in receiving disease control interventions compared to later IPs. As a result, both high contact rates and delayed application of interventions provided an opportunity during the initial phases of the outbreak periods for infectious birds to generate more and longer infection chains within flocks. This has important implications with respect to outbreak containment as it could result in large numbers of infected birds being present within such flocks prior to reporting if these flocks are involved in trade before movement restrictions is enforced, they can make a significant contribution to geographical spread of the virus.

Our results suggest that the estimates of within-flock $R_{0}$ for Period I are broadly comparable to those reported for HPAIV H5N1 infected chicken flocks from Thailand (e.g. 2.26-2.64, for infectious periods of 1 and 4 days respectively) [18]. Any observed differences might be due to differences in the quality of the control policies applied, or the population demographics and husbandry of flocks in each country. These factors are known to affect the 
underlying contact structure of a population which is an important driver of virus transmission [24]. In addition, the quality of the mortality data and the estimation methods used in the current study could have contributed to estimation bias and consequently to differences in $R_{0}$ estimates between both studies.

The findings of our study are also consistent with avian influenza vaccine trials that suggest that the use of vaccines is expected to reduce virus transmission and associated mortality among poultry by increasing the incubation period and reducing virus shedding [20,21]. Our results indicate that IPs reported during Period II develop lower mortality than their pre-vaccination counterparts. However, because farmers often use mortality as a flock health indicator this may contribute to the reduced ability of farmers and animal health workers to recognise the presence of disease. Other studies in unvaccinated populations have found that farmers recognize the abnormally high mortality resulting from HPAIV H5N1 approximately 5 days after infection [23].

Overall, our results showed that the mean within-flock $R_{0}$ of IPs from Period II was significantly lower compared to Period I - however, this reduction was not significantly below the threshold of transmission. Considering that IPs from Period II were classified as unvaccinated, this finding suggests that suboptimal vaccine coverage will lead to the re-emergence of outbreaks. Apart from issues related to the quality of protection provided by the vaccine, the overall effectiveness of the vaccination campaigns in target species is expected to be undermined by factors that deter farmers with commercial sized flocks from presenting their flocks for vaccination and operational issues for vaccine delivery. The former may be linked to the length of vaccine-withholding period and rumors concerning adverse reactions to the vaccine while the latter may be affected by issues such as the training and payment of vaccinators, the breakdown or spoilage of vaccine stocks and the fact that poultry husbandry is associated with rapid turn-over of at-risk populations. These factors have previously been documented in Vietnam and are likely to act as constraints to suboptimal vaccine coverage and consequently the effectiveness of the current vaccination campaigns [25]. These concerns are supported by the results of our multivariable analysis which indicate that commercial sized flocks (more than 50 head) have reduced odds of having within-flock $R_{0}$ estimates below unity. Therefore, in Vietnam, a control strategy based on nationwide HPAIV H5N1 vaccination campaigns should take into account the heterogeneities mentioned above [26]. This is particularly imperative when field evidence from other countries indicates that vaccination alone will not achieve disease elimination unless it is managed appropriately as part of a wider disease control strategy [27-31].

Our results should be interpreted considering the study's assumptions and limitations. Firstly, in our analyses, we assume that within-flock virus transmission will be halted in a scenario of total flock depopulation. However, if these flocks were left to develop further chains of infection (due to delays in disease reporting and depopulation) the within-flock $R_{0}$ estimate would be different. Secondly, our estimation method assumes a single introduction as the source of infection for an IP. It is known that pre-emptively culled farms were allowed to repopulate their flocks after a period of 60 days, but data on the proportion of farms which repopulated and the infection status of birds involved are unreliable. Similarly, there is no information with respect to the previous history of infection of these flocks and about the source leading to the current infection. Thirdly, we assume that the contact structure of flocks of similar species and size composition is constant, which in some situations may not be the case due to farm-to-farm variation in husbandry systems. Fourthly, we have assumed that all flocks included in the analysis were closed populations which, in the case of free-ranging flocks, may have led to an overestimation of the $R_{0}$ - however, the available data do not allow us to differentiate whether flocks included in the analysis were scavenging or not at the moment when they were reported infected.

The results of this study indicate that vaccination has protected the majority of poultry flocks against infection. However, our findings also provide evidence of the potential shortcomings of the current vaccine-based policy. Despite the control programme protecting the majority of farms as reflected in the very much reduced national incidence, the policy was not as effective in the flocks included in our analyses. To prevent similar cases, which are likely to become a continuing source of infection for poultry and humans, it is important to understand the factors behind the failure of the control policy in such flocks. In addition, we anticipate that if vaccination continues to be included as part of a sustainable disease control programme, efforts should be focussed on training farmers in disease prevention in addition to disease recognition, as the latter is likely to be compromised in a vaccinated population. Efforts must also be made to reduce operational delays in the implementation of disease control interventions after the recognition of the initial IP.

\section{Conclusions}

The results of this study show marked differences in the within-flock transmissibility of HPAIV H5N1 in Vietnam, 
before and after the introduction of a control policy that included vaccination. Our study showed:

- Within-flock mortality was lower in IPs reported after the introduction of a control policy that included vaccination compared to pre-vaccination IPs.

- Higher within-flock $R_{0}$ estimates were found in earlier phases of the outbreak periods examined.

- The mean within-flock $R_{0}$ in reported IPs was significantly lower in Period II (control based on depopulation plus vaccination) compared with Period I (control based on depopulation), but this reduction was not significantly below unity.

- After controlling for the effect of epidemic wave, commercial flocks ( $>50$ head) had reduced odds of having within-flock $R_{0}$ estimates below unity compared to flocks of smaller size ( $<50$ head).

Given the above, the observed effect is likely to be associated with the type and quality of disease control operations that were implemented during particular periods of the epidemic waves.

\section{Methods}

\section{Outbreaks of HPAIV H5N1 in poultry}

We analysed outbreak surveillance data on known infected flocks in Vietnam from two outbreak periods: one outbreak period from $1^{\text {st }}$ December 2005 to $29^{\text {th }}$ March 2005 (i.e. before the first systematic vaccination campaign - Period I) and another outbreak period from $16^{\text {th }}$ November 2006 to $7^{\text {th }}$ March 2007 (i.e. during the third and fourth nationwide systematic vaccination campaigns - Period II) (see section \#2 in Additional file 1). These periods reflect the second and fourth outbreak waves that occurred in the country since 2003/7 [4].The datasets used in the analyses only contained known affected flocks and were provided by the Epidemiology Division of the Department of Animal Health of the Ministry of Agriculture and Rural Development, Hanoi, Vietnam. All flocks included in the study were officially confirmed positive for HPAIV H5N1 virus by virus isolation and PCR at the National Centre of Veterinary Diagnostics (NCVD). In addition, the outbreak investigations conducted in relation to all flocks from Period II included in this analysis revealed that they had not been vaccinated [1].

\section{Estimation of the within-flock reproductive number, $\mathbf{R}_{0}$}

We estimated $R_{0}$ based on the general state-transition epidemic model adapted to the known properties of HPAIV H5N1 infection within a poultry flock. We applied the theory of moments of martingales to a general epidemic model informed by surveillance data from each infected flock included in the dataset (see section \#3 in
Additional file 1). The method was first described by Becker et al [32] and applied to infectious disease quantification studies in humans and animal populations [3335]. It has been described as a robust method for statistical estimation of the infection potential of a population when there is incomplete follow up of its infectious status $[36,37]$. The model formulation assumes a) uniform within-flock mixing, b) flocks homogeneity, and c) that an outbreak within an IP is a result of a single introduction. Finally, we estimated the mean within-flock $R_{0}$ for predefined species and size categories (see section \#2 in Additional file 1). The estimation procedures were implemented in the statistical software R [38].

\section{Standard statistical methods}

Comparisons between the mean within-flock $R_{0}$ in subsets of data were conducted using $t$-tests whenever data fulfilled the assumptions of normality and equal variances. Otherwise a non-parametric Kruskal-Wallis test was applied. One sample $t$-tests were performed to assess if the group mean within-flock $R_{0}$ was different from unity.

The statistical analysis leading to the identification of factors associated with within-flock $R_{0}$ estimates was carried out in two phases using 1 ) within-flock $R_{0}$ in a continuous scale outcome (Model 1) and 2) within-flock $R_{0}$ categorised into a threshold value (i.e. $R_{0}<1$ or $R_{0} \geq 1$ ) (Model 2). Firstly, flock species (categorized into duck flocks, mixed poultry species and chicken flocks), flock size (categorised into $<50$ head, 50-500 head, 500-1,500 head and $>1,500$ head) and epidemic wave (categorised into Period I and Period II) were screened using univariable linear regression (Model 1) and logistic regression (Model 2) based on a liberal $P$-value of 0.20 in the likelihood-ratio test. Secondly, all factors significant in the screening phase were considered for inclusion through a manual backward stepwise variable selection process in a multivariable linear regression (Model 1) and in a logistic regression (Model 2) analysis. The criterion for removal of risk factors was based on the likelihood ratio test with a significance level of $P>0.05$. Biologically meaningful first-order interaction terms were also tested for statistical significance. The goodness-of-fit of the final multivariable Model 1 was assessed by inspection of the coefficient of determination $\left(R^{2}\right)$ and for the final multivariable Model 2 was assessed by the Hosmer-Lemeshow goodness-of-fit test [39].All statistical analyses were conducted using the statistical software Stata/SE Version 9.2 (Stata Corporation) [40].

\section{Additional material}

Additional file 1 Supplementary technical information. 


\section{Authors' contributions}

RJSM conceived the study, conducted the analysis and wrote the manuscript. DUP supervised the study and critically revised the manuscript. JO critically reviewed the manuscript. All authors read and approved the final manuscript.

\section{Acknowledgements}

We are grateful for the valuable contribution of all senior officials and epidemiologists of the Department of Animal Health of The Ministry of Agriculture and Rural Development of Vietnam. Special thanks go to Dr. Van Nam and Dr. Dung Do for their hard work and dedication to HPAIV H5N1 control in Vietnam. We would like to thank the valuable contribution of Kate van Dooren for editing and proofreading the final manuscript. We would like to express our gratitude to the UK Department for International Development (DFID) for funding this work through the HPAl risk reduction project (GCP/UK/804/INT). Special thanks go to Dr. Jeff Gilbert and Davide Ferrardi of the FAO - Vietnam office.

\section{Author Details}

1The Royal Veterinary College, Department of Veterinary Clinical Sciences, Veterinary Epidemiology and Public Health Group, London, UK, Hawkshead Lane AL9 7TA, UK, 2 School of Population Health, University of Queensland, Edith Cavell Building, Herston Road, Herston QLD 4006, Australia and ${ }^{3}$ Food and Agriculture Organization of the United Nation, Pro-Poor Livestock Initiative, Vialle Del Terme de Caracalla, 00153 Rome, Italy

Received: 14 July 2009 Accepted: 5 June 2010

Published: 5 June 2010

\section{References}

1. Office International des Epizooties [http://www.oie.int/downld avian\%20influenza/A Al-Asia.htm]. Update of Highly Pathogenic Avian Influenza in Animals (Type $\mathrm{H} 5$ and $\mathrm{H} 7$ )

2. Minh PQ, Morris RS, Schauer B, Stevenson M, Benschop J, Nam HV, Jackson R: Spatio-temporal epidemiology of highly pathogenic avian influenza outbreaks in the two deltas of Vietnam during 2003-2007. Prev Vet Med 2009, 89(1-2):16-24.

3. Aly MM, Arafa A, Hassan MK: Epidemiological findings of outbreaks of disease caused by highly pathogenic H5N1 avian influenza virus in poultry in Egypt during 2006. Avian diseases 2008, 52(2):269-277.

4. Pfeiffer DU, Minh PQ, Martin V, Epprecht M, Otte MJ: An analysis of the spatial and temporal patterns of highly pathogenic avian influenza occurrence in Vietnam using national surveillance data. Vet $J$ 2007, 174(2):302-309.

5. Rushton J, Viscarra R, Bleiche EG, McLeod A: Impact of influenza outbreaks in the poultry sectors of five South east Asian countries (Cambodjia, Indonesia, Lao PDR, Thailand, Viet Nam) outbreak costs, responses and potential long term control. FAO, Rome, Italy; 2004:25.

6. Delquigny T, Edan M, Nguyen DH, Pham TK, Gautier P: Impact of Avian Influenza Epidemic and Description of the Avian Production in Vietnam. Rome, Italy: Food and Agriculture Organization of the United Nations; 2004:119.

7. ACl: The Economic Impact of Highly Pathogenic Avian Influenza Related Biosecurity Policies on the Vietnamese Poultry Sector. Bethesda, Maryland: Prepared for the Food and Agriculture Organization of the United Nations and the World Health Organization, Agrifood Consulting International; 2007:1013.

8. GSO: Socio-economic Impact of Avian Influenza. Hanoi: General Statistics Office - Vietnam; 2004.

9. World Health Organization: Cumulative Number of Confirmed Human Cases of Avian Influenza A/(H5N1) Reported to WHO. [http:// www.who.int/csr/disease/avian influenza/country/ cases table 201005 06/en/index.html].

10. Nguyen TD: The 2003-2004 H5N1 avian influenza outbreak in Vietnam. In The Threat of Pandemic Influenza: Are We Ready? Edited by: Knobler SL, Mack A, Mahmoud A, Lemon SM. Washington, DC: National Academy Press; 2005:130-140.

11. Taylor N, Do Huu D: An Analysis of Data generated by post-vaccination sero-monitoring and surveillance activities, following HPAI vaccination in Vietnam (2005-2006). Technical Report. FAO/DAH/MARD 2007:46.

12. Anderson RM, May RM: Population biology of infectious diseases: Part I. Nature 1979, 280(5721):361-367.
13. Diekmann O, Heesterbeek JAP: Mathematical Epidemiology of Infectious Diseases: Model Building, Analysis and Interpretation. Chicester, West Sussex: John Wiley \& Sons Ltd; 2000.

14. Ferguson NM, Cummings DA, Cauchemez S, Fraser C, Riley S, Meeyai A, lamsirithaworn S, Burke DS: Strategies for containing an emerging influenza pandemic in Southeast Asia. Nature 2005, 437(7056):209-214.

15. Longini IM Jr, Nizam A, Xu S, Ungchusak K, Hanshaoworakul W, Cummings DA, Halloran ME: Containing pandemic influenza at the source. In Science Volume 309. Issue 5737 New York, NY; 2005:1083-1087.

16. Savill NJ, St Rose SG, Keeling MJ, Woolhouse ME: Silent spread of H5N1 in vaccinated poultry. Nature 2006, 442(7104):757.

17. Stegeman A, Bouma A, Elbers AR, de Jong MC, Nodelijk G, de Klerk F, Koch $G$, van Boven M: Avian influenza A virus (H7N7) epidemic in The Netherlands in 2003: course of the epidemic and effectiveness of control measures. J Infect Dis 2004, 190(12):2088-2095.

18. Tiensin T, Nielen M, Vernooij H, Songserm T, Kalpravidh W Chotiprasatintara S, Chaisingh A, Wongkasemjit S, Chanachai K, Thanapongtham W, et al: Transmission of the Highly Pathogenic Avian Influenza Virus H5N1 within Flocks during the 2004 Epidemic in Thailand. Journal of Infectious Diseases 2007, 196:1679-1684.

19. Giesecke J: The epidemiology of vaccination. Modern Infectious Disease Epidemiology. Arnold 2nd edition. 2002:226-258.

20. Swayne DE: Principles for vaccine protection in chickens and domestic waterfowl against avian influenza: emphasis on Asian H5N1 high pathogenicity avian influenza. Annals of the New York Academy of Sciences 2006, 1081:174-181.

21. Bosch JC, De Jong MC, Franken P, Frankena K, Hage JJ, Kaashoek MJ, Maris-Veldhuis MA, Noordhuizen JP, Van der Poel WH, Verhoeff J, et al.: An inactivated gE-negative marker vaccine and an experimental gDsubunit vaccine reduce the incidence of bovine herpesvirus 1 infections in the field. Vaccine 1998, 16(2-3):265-271.

22. Giesecke J: Mathematical models for epidemics. Modern Infectious Disease Epidemiology. Arnold 2nd edition. 2002:119-132.

23. Yoon H, Park CK, Nam HM, Wee SH: Virus spread pattern within infected chicken farms using regression model: the 2003-2004 HPAl epidemic in the Republic of Korea. Journal of veterinary medicine 2005, 52(10):428-431.

24. Keeling MJ, Eames KT: Networks and epidemic models. J R Soc Interface 2005, 2(4):295-307.

25. Sims LD: Avian Influenza Emergency Recovery Project - Mission Report. FAO, Rome, Italy/World Bank; 2005:44.

26. Domenech J, Dauphin G, Rushton J, McGrane J, Lubroth J, Tripodi A, Gilbert J, Sims LD: Experiences with vaccination in countries endemically infected with highly pathogenic avian influenza: the Food and Agriculture Organization perspective. Revue scientifique et technique (International Office of Epizootics) 2009, 28(1):293-305.

27. Lee CW, Senne DA, Suarez DL: Effect of vaccine use in the evolution of Mexican lineage H5N2 avian influenza virus. J Virol 2004, 78(15):8372-8381

28. Villarreal $\mathrm{CL}$ : Control and eradication strategies of avian influenza in Mexico. Developments in biologicals 2006, 124:125-126.

29. Alexander DJ: Avian influenza viruses and human health. Developments in biologicals 2006, 124:77-84.

30. Capua I, Marangon S: The use of vaccination to combat multiple introductions of Notifiable Avian Influenza viruses of the $\mathrm{H} 5$ and $\mathrm{H} 7$ subtypes between 2000 and 2006 in Italy. Vaccine 2007, 25(27):4987-4995.

31. Capua I, Marangon S: The challenge of controlling notifiable avian influenza by means of vaccination. Avian diseases 2007, 51(1 Suppl):317-322.

32. Becker N: Analysis of Infectious Disease Data. In Monographs on Statistics and Applied Probability London: Chapman and Hall; 1989:139-174

33. Longini IM Jr, Halloran ME, Haber M, Chen RT: Measuring vaccine efficacy from epidemics of acute infectious agents. Statistics in medicine 1993, 12(3-4):249-263.

34. De Jong MC, Kimman TG: Experimental quantification of vaccineinduced reduction in virus transmission. Vaccine 1994, 12(8):761-766.

35. Mars MH, de Jong MC, Franken P, van Oirschot JT: Efficacy of a live glycoprotein E-negative bovine herpesvirus 1 vaccine in cattle in the field. Vaccine 2001, 19(15-16):1924-1930. 
36. Becker N: Statistical Challenges of Epidemics. In Epidemic Models:Their Structure and Relation to Data Edited by: Mollison D. Cambridge: Cambridge University Press; 1995:424.

37. Becker N: Martingale methods. In Analysis of Infectious Disease Data London: Chapman and Hall Ltd; 1989:224.

38. R Development Core Team R: A language and environment for statistical computing. Vienna, Austria R Foundation for Statistical Computing; 2004

39. Dohoo I, Martin W, Stryhn H: Logistic Regression. In Veterinary Epidemiological Research Prince Edward Island, Canada: AVC Inc; 2004:335-369.

40. StataCorp: Stata Statistical Software: Release 9. College Station, TX: StataCorp LP; 2005

\section{doi: 10.1186/1746-6148-6-31}

Cite this article as: Soares Magalhães et al., Evaluating the control of HPAIV H5N1 in Vietnam: virus transmission within infected flocks reported before and after vaccination BMC Veterinary Research 2010, 6:31

Submit your next manuscript to BioMed Central and take full advantage of:

- Convenient online submission

- Thorough peer review

- No space constraints or color figure charges

- Immediate publication on acceptance

- Inclusion in PubMed, CAS, Scopus and Google Scholar

- Research which is freely available for redistribution

Submit your manuscript at www.biomedcentral.com/submit
C) Biomed Central 